\title{
BMJ Open Statin-associated muscle symptoms (SAMS) in primary prevention for cardiovascular disease in older adults: a protocol for a systematic review and meta-analysis of randomised controlled trials
}

Zhen Zhou, ${ }^{1,2}$ Loai Albarqouni, ${ }^{3}$ Monique Breslin, ${ }^{1}$ Andrea J Curtis, ${ }^{4}$ Mark Nelson ${ }^{1}$

To cite: Zhou Z, Albarqouni L, Breslin M, et al. Statinassociated muscle symptoms (SAMS) in primary prevention for cardiovascular disease in older adults: a protocol for a systematic review and meta-analysis of randomised controlled trials. BMJ Open 2017;7:e017587. doi:10.1136/ bmjopen-2017-017587

- Prepublication history for this paper is available online. To view these files, please visit the journal online (http://dx.doi org/10.1136/bmjopen-2017017587).

Received 3 May 2017 Revised 18 August 2017 Accepted 23 August 2017

\section{(a) CrossMark}

${ }^{1}$ Menzies Institute for Medical Research, University of Tasmania, Hobart, Tasmania, Australia

${ }^{2}$ Anhui Medical University, Hefei, Anhui, China

${ }^{3}$ Faculty of Health Sciences and Medicine, Bond University, Gold Coast, Queensland, Australia ${ }^{4}$ Department of Epidemiology and Preventive Medicine, School of Public Health and Preventive Medicine, Monash University, Melbourne, Victoria, Australia

Correspondence to

Zhen Zhou;

zhen.zhou@utas.edu.au

\section{ABSTRACT}

Introduction Although statins are commonly used for prevention of cardiovascular disease, there is limited evidence about statin-related adverse effects in older people. Statin-related adverse events (AEs), especially the statin-associated muscle symptoms (SAMS), are the most common reasons for their discontinuation. Therefore, it is important to determine the risk of SAMS in the older population. We will undertake a systematic review and meta-analysis primarily focusing on the risk of SAMS and secondarily targeting myopathy, rhabdomyolysis, AEs and serious AEs, dropouts due to SAMS in run-in period, related permanent discontinuation rate of statins and creatine kinase level, among older people who received statins for primary prevention.

Methods and analysis This study has been developed according to the Preferred Reporting Items for Systematic Reviews and Meta-Analyses Protocols statement. We will include randomised controlled trials in which statin was compared with placebo with at least 1 year follow-up among older adults aged $\geq 65$. This review is an update of a Cochrane systematic review that included the articles published before 2012. Cochrane Central Register of Controlled Trials, Medline OvidSP and Embase electronic database searches will be performed to identify relevant articles, limiting the publication date from 1 January 2012 to 13 February 2017. There will be no language limitation. Two independent reviewers will screen titles and abstracts and full text in duplicate. Risk of bias and evidence quality will be assessed using the Cochrane Collaboration's tool and the Grading of Recommendations Assessment, Development and Evaluation approach, respectively. A meta-analysis using pooled data will be undertaken, if appropriate. We will also perform metaregression and subgroup analyses to identify sources of heterogeneity.

Ethics and dissemination This study is exempt from ethics approval due to the anonymous and aggregated data used. The outcomes will be disseminated by conference presentations and published in a peerreviewed journal.

Trial registration number CRD42017058436.
Strengths and limitations of this study

- To our knowledge, this study will be the first systematic review primarily exploring the risk of statin-associated muscle symptoms in older adults who received statins for primary prevention.

- The protocol has been developed following the Preferred Reporting Items for Systematic Reviews and Meta-Analyses-Protocols guideline.

- This study will contribute to strengthen the evidence base on the safety profile of statins by pooling the data from double-blind, placebo-controlled randomised controlled trials (RCTs).

- As few prior primary prevention studies have specifically targeted the elderly, most data on the occurrence of statin-associated muscle symptoms (SAMS) will be obtained from older subgroups in the selected RCTs.

- The lack of systematic collection of data on SAMS or adverse events (AEs) and the variability in definitions of SAMS and AEs may introduce potential ascertainment bias of outcomes.

\section{INTRODUCTION}

\section{Rationale}

Cardiovascular disease (CVD) is the leading cause of death globally. The WHO has estimated that 17.5 million people died from CVD in 2012, accounting for $31 \%$ of all global deaths. ${ }^{1}$ Age is the predominant risk factor for CVD with about $70 \%$ of adults older than 65 years having either coronary artery disease (CAD) or subclinical atherosclerosis. ${ }^{2}$ Moreover, older adults (aged $\geq 65$ years) account for more than $80 \%$ of the total CVD deaths. ${ }^{3}$ Thus, reducing mortality and morbidity of CVD in this age group is of paramount importance in reducing related cost and patient's disability. Additionally, according to the United Nations global demographic report, 
Box 1 Searching strategy designed for Embase database

1. exp hydroxymethylglutaryl coenzyme A reductase inhibitor/

2. hydroxymethylglutaryl ${ }^{*} . t w$.

3. HMG-CoA*tw.

4. (statin or statins).tw.

5. cerivastatin.tw.

6. fluvastatin.tw.

7. Iovastatin.tw.

8. atorvastatin.tw.

9. pravastatin.tw.

10. simvastatin.tw.

11. lipitor.tw.

12. baycol.tw.

13. lescol.tw.

14. mevacor.tw.

15. altocor.tw.

16. pravachol.tw.

17. lipostat.tw.

18. zocor.tw.

19. mevinolin.tw.

20. compactin.tw.

21. fluindostatin.tw.

22. rosuvastatin.tw.

23. dalvastatin.tw.

24. cranoc.tw.

25. canef.tw.

26. locol.tw.

27. lochol.tw.

28. leucol.tw.

29. lescol.tw.

30. monacolin.tw.

31. medostatin.tw.

32. mevinacor.tw.

33. livalo.tw.

34. pitava.tw.

35. pitavastatin.tw.

36. pravasin.tw.

37. mevalotin.tw.

38. gerosim.tw.

39. lipex.tw.

40. zenas.tw.

41. crestor.tw

42. meglutol.tw.

43. or/1-42

44. exp cardiovascular disease/

45. cardio*.tw.

46. cardia*.tw.

47. heart*.tw.

48. coronary*.tw.

49. angina*.tw.

50. hyperlipidemia/

51. exp cholesterol/

52. exp lipid blood level/

53. hyperlipid*.tw.

54. hypercholesterol*.tw.

55. cholesterol*.tw.

56. hypercholester?emia*.tw.

57. hyperlip?emia*.tw.

58. triglycerid ${ }^{*}$.tw.

59. hypertriglycerid?emia*.tw. 


\section{Box 1 Continued}
60. hyperlipoprotein?emia*.tw.
61. LDL.tw.
62. HDL.tW.
63. 0 r/44-62
64. 43 and 63
65. limit 64 to $y r=" 2012$-Current'
66. (random $\$$ or placebo $\$$ or single blind $\$$ or double blind $\$$ or triple blind $\$$ ).ti,ab.
67. retracted article/
68. 66 or 67
69. (animal\$ not human\$). sh, hw.
70. (book or conference paper or editorial or letter or review).pt. not exp randomized controlled trial/
71. (random sampl\& or random digit\$ or random effect\$ or random survey or random regression).ti,ab. not exp randomized controlled trial/
72. 69 or 70 or 71
73. 68 not 72

people aged 60 years or more represented $12.5 \%$ of the global population in 2015 and this will increase to an estimated $16.7 \%$ in $2030 .{ }^{4}$ This will undoubtedly increase government expenditure on healthcare. On a population basis, primary prevention is very important for CVD prevention as healthy people represent the largest proportion of the general population. Therefore, the greatest potential for reduction in major adverse cardiovascular events (MACE) resides in this population. ${ }^{5}$

Statins (hydroxy-methyl-glutaryl-coenzyme A reductase inhibitors) have been shown to reduce MACE and mortality-in both middle-aged and older adults. ${ }^{67}$ The

\section{Box 2 Characteristics of included articles}

Method: type of study.

- Participants: sample size; country; age range (mean $\pm S D$ ); proportion of females; baseline low-density lipoprotein level; risk level of cardiovascular disease; any comorbidity such as diabetes or hypertension; loss to follow-up.

- Interventions: the name, dose and frequency of statins; comparison group; intervention in run-in phase; duration of follow-up and run-in phase.

- Outcomes: the primary outcome is statin-associated muscle symptoms (SAMS); The secondary outcomes are myopathy, rhabdomyolysis, adverse events (AEs), serious AEs, dropouts due to SAMS in run-in phase, total permanent discontinuations of statins and discontinuations due to AEs and especially muscular problems and a creatine kinase level $\geq 5$ times upper limit of normal.

Notes: whether trials stop prematurely; conflicts of interest

Box 3 Seven domains involved in Cochrane assessment tool for risk of bias

Random sequence generation (selection bias)

- Allocation concealment (selection bias)

- Blinding of participants and personnel (performance bias)

- Blinding of outcome assessment (detection bias)

- Incomplete outcome data (attrition bias)

- Selective reporting (reporting bias)

Other sources of bias (other bias) beneficial effect of statins on survival is evident 1-2 years after commencing statin treatment. ${ }^{8}$ A meta-analysis of observational studies found that about half of patients initiated on statins discontinued their medication within a year. Taking statins for primary prevention was determined to be a main predictor of non-adherence. ${ }^{9}{ }^{10}$ Only one in four people aged over 65 years was adherent to statins after 2 years of commencing statin for primary prevention. ${ }^{8}$ Additionally, statin users aged over 70 years have lower adherence rates than middle-aged adults (50-69 years).$^{10}$ While there are various reasons for statin discontinuation, two-thirds of the patients reported statin-related adverse effects as the reason. ${ }^{11}$ Older individuals are more vulnerable to adverse effects of drugs compared with younger individuals due to their physical deconditioning (decreased muscle mass and poorer renal and hepatic clearance), multiple morbidity and polypharmacy. ${ }^{12}{ }^{13}$ Recognised statin-associated adverse effects include musculoskeletal dysfunction, hepatotoxicity, new-onset type 2 diabetes and some other rare but significant adverse effects such as cancer, kidney disease and cataract. ${ }^{14-16}$ Of these, statin-associated muscle symptoms (SAMS), generally defined as all muscle-related complaints such as muscle pain or aching (myalgia), tenderness, stiffness, cramp and weakness, are the most commonly encountered adverse events (AEs) both in clinical studies and daily clinical practice.$^{17}$ It is estimated that more than 1.5 million people per year experience SAMS. ${ }^{18}$ Of note, myopathy and rhabdomyolysis, which accompanied with high creatine kinase (CK) level, are two severe statin-related musculoskeletal diseases that rarely happen but are potentially life threatening.

Statins are recommended to be initiated during early middle age for people with clinical atherosclerotic cardiovascular disease (ASCVD) and high estimated 10-year ASCVD risk due to the well-documented benefits in middle-aged adults. ${ }^{2} 1920$ Although the beneficial effects of statins in the middle and younger adults are well documented, the older individuals have been under-represented in statin clinical trials, particularly those focusing on primary prevention and drug safety. ${ }^{21} 22$ 
Table 1 The scoring system provided by GRADE Working Group on assessing the quality of the evidence

\section{Type of evidence}

Initial score based on $+4 \quad$ RCTs/SR of RCTs,+/-othertypes of evidence

type of evidence $\quad+2 \quad$ Observational evidence (eg, cohort, case-control)

$\begin{array}{ll}\text { Quality } & \text { Blinding and allocation process } \\ & \text { Follow-up and withdrawals } \\ & \text { Sparse data } \\ & \text { Other methodological concerns (eg, incomplete reporting, subjective outcomes) } \\ \text { Score } & \text { Score } 0,-1,-2,-3 \text { represents 'no problems', problem with one element', 'problem with two elements', } \\ & \text { 'problem with three elements', respectively. }\end{array}$

\section{Consistency}

Based on Degree of consistency of effect between or within studies

Score $\quad+1 \quad$ Evidence of dose response across or within studies (or inconsistency across studies is explained by a dose response); also one point added if adjustment for confounders would have increased the effect size

$0 \quad$ All/most studies show similar results

$-1 \quad$ Lack of agreement between studies (eg, statistical heterogeneity between RCTs, conflicting results)

\begin{tabular}{|c|c|c|}
\hline \multicolumn{3}{|l|}{ Directness } \\
\hline Based on & \multicolumn{2}{|r|}{ The generalisability of population and outcomes from each study to our population of interest } \\
\hline Score & \multicolumn{2}{|r|}{$\begin{array}{l}\text { Score } 0,-1,-2 \text { represents 'population and outcomes broadly generalisable', 'problem with one element', } \\
\text { 'problem with two or more elements', respectively. }\end{array}$} \\
\hline \multicolumn{3}{|l|}{ Effect size } \\
\hline Based on & \multicolumn{2}{|c|}{ The reported OR/RR/HR for comparison } \\
\hline \multirow[t]{3}{*}{ Score } & 0 & Not all effect sizes $>2$ or $<0.5$ and significant; or if $O R / R R / H R$ not significant \\
\hline & +1 & Effect size $>2$ o $r<0.5$ for all studies/meta-analyses included in comparison and significant \\
\hline & +2 & Effect size $>5$ or $<0.2$ for all studies/meta-analyses included in comparison and significant \\
\hline
\end{tabular}

GRADE, Grading of Recommendations Assessment, Development and Evaluation; RCTs, randomised controlled trials; SR, systematic review; $\mathrm{OR}$, odds ratio; $\mathrm{RR}$, relative risk; $\mathrm{HR}$, hazard ratio.

There is still no definitive indication of statin therapy in the low-risk elderly due to a lack of evidence from clinical studies or meta-analyses (cited in Class IIb and Level of evidence in European Society of Cardiology guidelines) and making work more challenging in routine clinical practice. ${ }^{19}$ To our knowledge, one meta-analysis indicated that there is no excess risk of myopathy in older adults who received statins for both primary and secondary prevention, while no prior systematic review has ever primarily investigated the risk of SAMS among older people who received statins for only primary prevention. ${ }^{23}$ Clearly, studies are required, both randomised controlled trials (RCTs) (such as StaREE: Statins in Reducing Events in the Elderly, which is an ongoing blind, placebo-controlled clinical trial of statin therapy in primary prevention elderly: NCT02099123) and meta-analyses to provide evidence on drug safety of statin therapy prescribed in older adults for primary prevention of CVD and further to facilitate optimal prescribing and management approaches to minimise the side effects.

\section{Objectives}

We will primarily determine the risk of SAMS among older people ( $\geq 65$ years) who have received statins for primary prevention. In addition, we will determine the risk of myopathy, rhabdomyolysis, the AEs and serious adverse events (SAEs), the number of dropouts due to SAMS in run-in period, total permanent discontinuations (results from all cause) and permanent discontinuations related to AEs and specifically, muscular problems, patients with a CK level $\geq 5$ times upper limit of normal (ULN) among the same subpopulation group.

\section{METHODS}

This study has been designed and developed according to the Preferred Reporting Items for Systematic Reviews and Meta-Analyses Protocols statement. ${ }^{24}$ The protocol was registered with the International Prospective Register of Systematic Reviews (registration no CRD42017058436). 


\section{Eligibility criteria}

Studies will be selected according to the criteria outlined below.

\section{Study design}

We will include double-blind, randomised, placebo controlled trials. We will exclude RCTs with an open comparator, non-randomised controlled trials and observational studies (eg, cohort studies).

\section{Participants}

We will include studies with participants who do not have established CVD. In addition, eligible studies should have restricted inclusion to participants aged 65 years or older or have provided a subanalysis for this group. Also eligible are studies in which the authors are willing to share patient-level data so that this age restriction can be imposed as part of this analysis. We will exclude studies that included patients with specialised pre-existing disease such as cancer, hypothyroidism, acute infection, chronic renal disease, HIV, post-transplantation or any other acute illness that might affect the study outcomes.

\section{Interventions}

We will include studies with a statin (eg, atorvastatin, fluvastatin, lovastatin, pravastatin, rosuvastatin or simvastatin) as the intervention and placebo as comparator. We will exclude studies using cerivastatin as a statin since it was withdrawn from the market due to a high risk of rhabdomyolysis. ${ }^{25}$ We will also exclude the studies in which the combination of any other lipid-lowering medications and statin was used as an intervention.

\section{Timing}

Studies should have a follow-up time of at least of 1 year to be eligible for inclusion.

\section{Outcomes}

Studies should report at least one of the following outcomes to be eligible: SAMS, myopathy, rhabdomyolysis, AEs, SAEs, dropouts due to SAMS in run-in period, total permanent discontinuations of statins and discontinuations due to AEs or muscular problems, elevation in CK defined as a CK level $\geq 5$ times ULN.

\section{Language}

There will be no restrictions by language of publication.

\section{Search strategy}

This systematic review will be an update of a relevant Cochrane systematic review in which the articles published prior to 2012 were identified. ${ }^{26}$ This Cochrane review included a larger population because it was aimed at statin therapy for primary prevention across all age groups. For the articles published before 1 January 2012, we will make a further assessment by selecting the eligible articles from the articles included in this Cochrane review. For the articles published from 1 January 2012 to 13 February 2017, we will establish a new search using the same search strategy listed in the Cochrane review apart from the publication date. The Cochrane Central Register of Controlled Trials, Medline OvidSP and Embase databases will be performed to identify any relevant articles published. A combination of medical subject headings terms (such as 'Hydroxymethylglutaryl-CoA Reductase Inhibitors', 'Hyperlipidemias', 'Cholesterol', 'Cardiovascular Diseases') and related free text terms (such as 'hydroxymethylglutaryl*', 'cardio*', 'hyperlipid*', 'cholesterol*') will be used. Term 'random\$ or placebo\$ or single blind \$ or double blind\$ or triple blind\$' will be used to filter the RCTs. Furthermore, we will hand search the relevant review articles to help retrieve all eligible trials. A complete Embase search strategy is included in box 1 . The search strategy will be adapted to the other databases.

\section{Selection process}

Records from three databases will be exported to Endnote V.X8 (Bld 10063). After removing duplicate records, two review authors ( $\mathrm{ZZ}$ and $\mathrm{LA}$ ) will independently screen the title and abstract against the eligibility criteria. Discrepancies will be resolved by consensus after discussion. A recommendation will be given by the third author (MN) if consensus cannot be reached. The full text for titles and abstracts that meet the inclusion criteria will be obtained. Two review authors (ZZ and LA) will independently screen the full text of identified records and record the reasons for excluding trials.

\section{Data extraction}

We will use a standardised data extraction sheet to extract data from each included study. Data extracted will include study characteristics, methodology, intervention details and all relevant outcomes (see box 2 for extracted data). We will contact study authors to obtain missing data.

\section{Outcomes and prioritisation}

1. The primary outcome is SAMS. Of note, we will only consider symptomatic muscular symptoms that matter to the participant, which included myalgia (muscle pain), muscle weakness, stiffness, tenderness and cramp. ${ }^{27}$ Asymptomatic, such as haematological index and pathological alteration will not be taken into account as they are less significant to participants who care more about symptomatic problems that compromise their life quality.

2. Our secondary outcome will measure myopathy (defined as SAMS with CK $>10 \times \mathrm{ULN})^{17}$; rhabdomyolysis (defined as SAMS with CK $>40 \times$ ULN when accompanied with renal impairment and/ or myoglobinuria) ${ }^{17}$; AEs; SAEs (defined as adverse experiences that were considered serious including life threatening, causing death or a permanent disability or incapacity, resulting in or prolonging hospitalisation ${ }^{28}$ ); dropouts due to SAMS in runin period; Total permanent discontinuations to statins and permanent discontinuations to statins 
due to adverse effects-related and specifically, muscular problem-related reasons in RCTs, compared with placebo group. Additionally, we will also examine the incidence of high CK level $\geq 5 \times \mathrm{ULN}$ as this is a meaningful predictor of myotoxicity.

\section{Quality assessment (risk of bias of individual studies)}

Two independent review authors (ZZ and LA) will assess the risk of bias of all included studies separately. We will use the Cochrane Collaboration tool for assessing the risk of bias, which include seven specific domains ${ }^{29}$ (see box 3 for the seven assessment criteria). Each domain will be assessed and categorised as 'Low risk', 'High risk' or 'Unclear risk' of bias. Two figures will be generated using RevMan in our article. One is a 'Risk of bias graph' figure that will present the proportion of studies with each of the assessments for each entry in the tool. Another is a 'Risk of bias summary' figure that will show all assessments in a cross-tab for each study. ${ }^{29}$

\section{Data synthesis and analysis}

For all primary and secondary outcomes in our study, as dichotomous data, relative risks (RRs) with 95\% confidence intervals (CIs) will be calculated for the pooled effects. Statistical analysis of outcomes will be based on 'intention-to-treat' principle. The statistical heterogeneity between individual studies as measured by $\mathrm{I}^{2}$ test will be reported. ${ }^{30} \mathrm{I}^{2}$ values of $30 \%$ to $60 \%, 50 \%$ to $90 \%, 75 \%$ to $100 \%$ may indicate moderate, substantial and considerable heterogeneity, respectively. ${ }^{29}$ In view of the variation of the follow-up duration, the type and the dosage of statins and the assessment methods on AEs profile across the included RCTs, the random-effects model (DerSimonian and Laird method) will be fitted in our study. ${ }^{31}{ }^{32}$ We will not perform a meta-analysis if the heterogeneity is substantial or there is a lack of data for any comparison; a narrative, qualitative summary will be done. Where significant heterogeneity is present, meta-regression and subgroup analyses for the primary outcome will also be conducted to identify the sources of heterogeneity according to the following covariates: statin solubility (hydrophilic or lipophilic), dose (standard or intensive) and type of statins, study duration, gender and different comorbidities of subjects (such as diabetes and hypertension). In addition to this, a leave-one-out sensitivity analysis will be conducted by iteratively removing one study at a time to assess the impact of each study. STATA statistical software V.14.2 (Stata/SE for windows) will be used for all the analyses.

\section{Publication bias assessment}

Publication bias will be assessed using the funnel plot test if the number of articles is sufficient $(>10)$. Asymmetry identified in the funnel plot implies possible publication bias. ${ }^{29}$ In addition to this, contour-enhanced meta-analysis funnel plots will be conducted to distinguish publication bias from other causes of asymmetry. ${ }^{33}$ The Egger's regression-intercept test will also be performed to identify publication bias. ${ }^{34}$ The funnel plots and Egger's regression test will be generated by STATA.

\section{Confidence in cumulative evidence}

The scoring system (table 1) provided by the Grading of Recommendations Assessment, Development and Evaluation (GRADE) Working Group will be used to assess the quality of the evidence. After summarising the score of each item in GRADE, the final score will be categorised to four levels: high ( $\geq 4$ points), moderate (three points), low (two points), very low ( $\leq 1$ point) ${ }^{35}$

Contributors MN is a guarantor. ZZ and LA designed the study and drafted the protocol. MB provided the statistics support. AJC and MN provided content expertise, checked the protocol and gave feedback. All the authors approved the final manuscript after rigorous review.

Competing interests None declared.

Provenance and peer review Not commissioned; externally peer reviewed.

Open Access This is an Open Access article distributed in accordance with the Creative Commons Attribution Non Commercial (CC BY-NC 4.0) license, which permits others to distribute, remix, adapt, build upon this work non-commercially, and license their derivative works on different terms, provided the original work is properly cited and the use is non-commercial. See: http://creativecommons.org/ licenses/by-nc/4.0/

(c) Article author(s) (or their employer(s) unless otherwise stated in the text of the article) 2017. All rights reserved. No commercial use is permitted unless otherwise expressly granted.

\section{REFERENCES}

1. Cardiovascular diseases (CVDs) Fact Sheet. World Health Organization 2016. http://www.who.int/mediacentre/factsheets/ fs317/en/

2. Catapano AL, Reiner Z, De Backer G, et al. ESC/EAS Guidelines for the management of dyslipidaemias: the task Force for the management of dyslipidaemias of the European Society of Cardiology (ESC) and the European Atherosclerosis Society (EAS). Atherosclerosis 2011;217:3-46.

3. Roger VL, Go AS, Lloyd-Jones DM, et al. Heart disease and stroke statistics-2012 update: a report from the American Heart Association. Circulation 2012;125:e2-220.

4. World population ageing 2015. United Nations $2015 \mathrm{http} / / / \mathrm{www}$. un.org/en/development/desa/population/publications/pdf/ageing/ WPA2015 Report.pdf

5. The world health report. Chapter 6. World Health Organization. http:// www.who.int/whr/2002/chapter6/en/index1.html

6. Shepherd J, Blauw GJ, Murphy MB, et al. Pravastatin in elderly individuals at risk of vascular disease (PROSPER): a randomised controlled trial. Lancet 2002;360:1623-30.

7. Ridker PM, Danielson E, Fonseca FA, et al. Rosuvastatin to prevent vascular events in men and women with elevated C-reactive protein. N Engl J Med 2008;359:2195-207.

8. Jackevicius CA, Mamdani M, Tu JV. Adherence with statin therapy in elderly patients with and without acute coronary syndromes. JAMA 2002;288:462-7.

9. Lemstra M, Blackburn D, Crawley A, et al. Proportion and risk indicators of nonadherence to statin therapy: a meta-analysis. Can $J$ Cardiol 2012;28:574-80.

10. Mann DM, Woodward M, Muntner P, et al. Predictors of nonadherence to statins: a systematic review and meta-analysis. Ann Pharmacother 2010;44:1410-21.

11. Maningat P, Gordon BR, Breslow JL. How do we improve patient compliance and adherence to long-term statin therapy? Curr Atheroscler Rep 2013;15:291.

12. Wilmot KA, Khan A, Krishnan S, et al. Statins in the elderly: a patientfocused approach. Clin Cardiol 2015;38:56-61.

13. Hilmer S, Gnjidic D. Statins in older adults. Aust Prescr 2013;36:79-82.

14. Silva MA, Swanson AC, Gandhi PJ, et al. Statin-related adverse events: a meta-analysis. Clin Ther 2006;28:26-35.

15. Saxon DR, Eckel RH. Statin intolerance: a literature review and management strategies. Prog Cardiovasc Dis 2016;59:153-64. 
16. Mancini GB, Baker S, Bergeron J, et al. Diagnosis, prevention, and management of statin adverse effects and intolerance: Canadian Consensus Working Group Update (2016). Can J Cardiol 2016;32(7 Suppl):S35-65.

17. Stroes ES, Thompson PD, Corsini A, et al. Statin-associated muscle symptoms: impact on statin therapy-European Atherosclerosis Society Consensus Panel Statement on Assessment, Aetiology and Management. Eur Heart J 2015;36:1012-22.

18. Sathasivam S, Lecky B. Statin induced myopathy. BMJ 2008;337:a2286.

19. Reiner Z, Catapano AL, Backer GD, et al. ESC/EAS Guidelines for the management of dyslipidaemias: the task force for the management of dyslipidaemias of the European Society of Cardiology (ESC) and the European Atherosclerosis Society (EAS). Atherosclerosis 2011;217(Suppl 1):S1-44

20. Stone NJ, Robinson JG, Lichtenstein AH, et al. 2013 ACC/ AHA guideline on the treatment of blood cholesterol to reduce atherosclerotic cardiovascular risk in adults: a report of the American College of Cardiology/American Heart Association Task Force on Practice Guidelines. Circulation 2014;129(25 Suppl 2):S1-45

21. Konrat C, Boutron I, Trinquart L, et al. Underrepresentation of elderly people in randomised controlled trials. the example of trials of 4 widely prescribed drugs. PLoS One 2012;7:e33559.

22. Gurwitz JH, Go AS, Fortmann SP. Statins for primary prevention in older adults: uncertainty and the need for more evidence. JAMA 2016;316:1971-2.

23. Iwere RB, Hewitt J. Myopathy in older people receiving statin therapy: a systematic review and meta-analysis. $\mathrm{Br} J$ Clin Pharmacol 2015;80:363-71.
24. Moher D, Shamseer L, Clarke M, et al. Preferred reporting items for systematic review and meta-analysis protocols (PRISMA-P) 2015 statement. Syst Rev 2015;4:1.

25. Furberg CD, Pitt B. Withdrawal of cerivastatin from the world market. Curr Control Trials Cardiovasc Med 2001;2:205-7.

26. Taylor FC, Huffman M, Ebrahim S. Statin therapy for primary prevention of cardiovascular disease. JAMA 2013;310:2451-2.

27. Thompson PD, Clarkson PM, Rosenson RS, et al. An assessment of statin safety by muscle experts. Am J Cardiol 2006;97:S69-76.

28. World Health Organization. Definitons. http://www.who.int/medicines/ areas/quality_safety/safety_efficacy/trainingcourses/definitions.pdf.

29. Higgins J, Green S. Cochrane handbook for systematic reviews of interventions version 5.1.0. The Cochrane collaboration $2011 \mathrm{http}: / /$ handbook-5-1.cochrane.org

30. Higgins JP, Thompson SG, Deeks JJ, et al. Measuring inconsistency in meta-analyses. BMJ 2003;327:557-60.

31. DerSimonian R, Laird N. Meta-analysis in clinical trials. Control Clin Trials 1986;7:177-88.

32. Borenstein M, Hedges LV, Higgins JP, et al. A basic introduction to fixed-effect and random-effects models for meta-analysis. Res Synth Methods 2010:1:97-111.

33. Peters JL, Sutton AJ, Jones DR, et al. Contour-enhanced metaanalysis funnel plots help distinguish publication bias from other causes of asymmetry. J Clin Epidemiol 2008;61:991-6.

34. Egger M, Davey Smith G, Schneider M, et al. Bias in meta-analysis detected by a simple, graphical test. BMJ 1997;315:629-34.

35. Guyatt G, Oxman AD, Akl EA, et al. GRADE guidelines: 1 . Introduction-GRADE evidence profiles and summary of findings tables. J Clin Epidemiol 2011;64:383-94. 